\title{
OTher Voices
}

\section{The Death Debates: A Call for Public Deliberation}

BY DAVID RODRÍGUEZ-ARIAS AND CARISSA VÉLIZ

T he language used to talk about death determination has been repeatedly reformulated. Examples include "brain death," to describe the condition of patients who were in "coma dépassé"; 1 consecutive adjustments in the language used to refer to the practices known early on as "non-heart-beating donation" (later as "donation after cardiac death," then as "donation after circulatory death," and then finally as "donation after circulatory determination of death" $)$; and proposals to replace "irreversible cessation" with "permanent cessation" to support controlled donation after circulatory determination of death (DCDD) protocols. ${ }^{3}$ Despite objections, these changes to language have entered or are entering into academic jargon, professional guidelines, and international regulations. The language seems, in part, to promote organ donation but also helps physicians formally avoid violations of the dead donor rule. ${ }^{4}$

Since the early 1980s, James Bernat has played a leading role in shaping both scholarly discourse and policy-making about death determination and organ procurement. His scientific contributions may be indirectly responsible for having extended the life expectancy and the quality of life of thousands of people around the world. However, the conceptual reformulations he and others have proposed have had intellectual and practical costs. Critics argue that linguistic gerrymandering, semantic contortions, and legal fictions reveal more concern for pragmatic goals than for truth. ${ }^{5}$ The effort also creates confusion among the public, the media, and even health professionals. ${ }^{6}$ It also raises practical objections concerning the respect for and protection of donors and their families.

David Rodríguez-Arias and Carissa Véliz, "The Death Debates: A Call for Public Deliberation," Hastings Center Report 43, no. 6 (2013): 34-35. DOI: $10.1002 /$ hast.232
In this issue of the Report, Bernat proposes an innovative and sophisticated distinction to justify the introduction of permanent cessation as a valid substitute standard for irreversible cessation in death determination. He differentiates two approaches to conceptualizing and determining death: the biological concept and the prevailing medical practice standard. While irreversibility is required by the biological concept, the weaker criterion of permanence, he claims, has always sufficed in the accepted standard medical practice to declare death. Bernat argues that the medical practice standard may be acceptable on the ground that proving circulatory or brain permanence is sufficient to assure complete accuracy for death diagnosis.

We doubt that permanence has been and in practice is sufficient for declaring death. In fact, we suspect that Bernat himself finds it insufficient. Why else does he discourage the use of extracorporeal membrane oxygenation in DCDD? Donors who are declared dead according to the permanence standard may recover neurological functions, including consciousness, when ECMO is introduced to preserve their organs. ${ }^{7}$ To prevent that, a balloon is customarily inserted to occlude the aorta and to restrict circulation to the abdomen. Such an intervention has been recommended to ensure that permanent loss of neurological function becomes irreversible ${ }^{8}$ and to avoid the disturbing risk —-hypothetical though it might be —of enabling first-person experiences of vital organ retrieval. Rejecting the use of ECMO is evidence, in our view, that one is uncomfortable with permanence alone.

Bernat also claims that irreversibility would produce unjustified harms to patients and their family members with no countervailing benefits. It is somewhat paradoxical to claim that a stronger criterion for certifying death would entail less protection than a weaker one. By itself, calling donors "dead" by the "permanence" standard when they have not yet irreversibly lost the capacity for 


\section{Not letting society participate in debates about the dead donor rule could be seen as a form of paternalistic expertocracy.}

consciousness hardly increases their chances of getting appropriate protection and respectful care. In fact, it might have the opposite effect, as doctors are not supposed to sedate cadavers or require their explicit consent to initiate certain interventions intended strictly to preserve their organs. ${ }^{9}$ If the objective is to respect and protect donors, wouldn't it be better to make sure they had given consent for donation and that they are beyond harm? ${ }^{10}$

Bernat overlooks what seems to us the most compelling reason to support the permanence standard: to promote DCDD organ procurement without violating the dead donor rule. Bernat has claimed elsewhere that "violating the DDR is misguided and will lead fearful patients to lose trust in physicians and confidence in the organdonation system and will result in an overall decline in organ donation." 11 In truth, if we are to continue with organ donation while honoring the DDR, there is no choice to be made between permanence and irreversibility: by the time biological death is a certainty in a DCDD protocol, the organs have deteriorated too much for transplantation. The real choice is between "respecting" the dead donor rule (by bending the criteria of death) and devising more sophisticated and transparent ways of protecting patients (for example, acknowledging that donors may not be fully dead when organs are retrieved and using anaesthesia for organ retrieval with their consent).

Shaping language in a way that hides the normative questions at stake undercuts public understanding. Lay people are less likely to debate claims that experts present as simple "facts" ("DCDD donors are dead") rather than as normative claims. This creates a concern about the public legitimacy of deceased organ donation: invoking the interest society has in not violating the dead donor rule without letting society participate in these debates could be considered a form of paternalistic expertocracy.

Bernat claims that any policy solution to this debate "should engender broad support among health professionals and the public by being conceptually coherent, physiologically plausible, and socially acceptable." $\mathrm{He}$ argues that, given the lack of public outcry, "death declaration at permanent cessation has been well accepted by society," even though "the public is probably mostly unaware of the practice." We believe that the absence of a public outcry can hardly be considered evidence of societal acceptance for a position that is surrounded by complexity, confusion, and lack of transparency. ${ }^{12}$

The topic requires public deliberation: processes to survey people's opinions and mechanisms to channel their opinions into policy-making. What is at stake is the nature of our society. Do we want an expertocracy, in which an enlightened few design policies for the greater good of the majority and exploit the lack of public knowledge to achieve compliance? Or a deliberative democracy, where transparency is nonnegotiable and people have the right not only to know what happens behind the expert curtains but also to voice their views on public policies and to press for their will to be carried out?

1. Ad Hoc Committee of the Harvard Medical School to Examine the Definition of Brain Death, "A Definition of Irreversible Coma: Report of the Ad Hoc Committee of the Harvard Medical School to Examine the Definition of Brain Death," Journal of the American Medical Association 205 (1968): 337-40.

2. J. L. Bernat, "The Debate over Death Determination in DCD," Hastings Center Report 40, no 3 (2010): 3.

3. K. G. Munjal et al., "A Rationale in Support of Uncontrolled Donation after Circulatory Determination of Death," Hastings Center Report 43, no. 1 (2013): 19-26.

4. D. Rodríguez-Arias, M. J. Smith, and N. M. Lazar, "Donation after Circulatory Death: Burying the Dead Donor Rule," American Journal of Bioethics 11, no. 8 (2011): 36-43.

5. R. M. Arnold and S. J. Youngner, "The Dead Donor Rule: Should We Stretch It, Bend It, or Abandon It?" Kennedy Institute of Ethics Journal 3, no. 2 (1993): 263-78; D. A. Shewmon, "Brain Death: Can It Be Resuscitated?" Hastings Center Report 39, no. 2 (2009): 18-24; S. K. Shah, R. D. Truog, and F. G. Miller, "Death and Legal Fictions," Journal of Medical Ethics 37, no. 12 (2011): 719-22; D. W. Brock, "Truth or Consequences: The Role of Philosophers in Policy-making," Ethics 97, no. 4 (1987): 786-91.

6. L. A. Siminoff, C. Burant, and S. J. Youngner, "Death and Organ Procurement: Public Beliefs and Attitudes," Social Sciences and Medicine 59, no. 11 (2004): 2325-34; A. Daoust and E. Racine, "Depictions of 'Brain Death' in the Media: Medical and Ethical Implications," Journal of Medical Ethics (2013), doi:10.1136/ medethics-2012-101260 (epub ahead of print); Rodríguez-Arias et al., "One or Two Types of Death? Attitudes of Health Professionals towards Brain Death and Donation after Circulatory Death in Three Countries," Medicine, Health Care, and Philosophy 16, no. 3 (2011): 457-67.

7. Bernat et al., "The Circulatory-Respiratory Determination of Death in Organ Donation."

8. S. P. Wall et al., "Derivation of the Uncontrolled Donation after Circulatory Determination of Death Protocol for New York City," American Journal of Transplantation 11, no. 7 (2011): 1417-26.

9. J. F. Childress, "Organ Donation after Circulatory Determination of Death: Lessons and Unresolved Controversies," Journal of Law, Medicine and Ethics 36, no. 4 (2008): 610, 766-71.

10. F. G. Miller and R. D. Truog, Death, Dying, and Organ Transplantation: Reconstructing Medical Ethics at the End of Life (New York: Oxford University Press, 2011).

11. J. L. Bernat, "Life or Death for the Dead-Donor Rule?," New England Journal of Medicine 369 (2013): 1289-91.

12. A. R. Joffe et al., "Donation after Cardiocirculatory Death: A Call for a Moratorium Pending Full Public Disclosure and Fully Informed Consent," Philosophy, Ethics, and Humanities in Medicine 6, no. 17 (2011), doi:10.1186/1747-5341-6-17. 